avances

en ciencias e ingenierías

II Simposio
Latinoamericano de Aplicaciones Nucleares

en la Agricultura

\section{Aplicación de rayos X para la obtención de mutantes ornamentales en Salvia coccinea}

\author{
Verónica Bugallo ${ }^{1,2}$, Gabriela Facciuto ${ }^{2}$, Vanina Brizuela ${ }^{3}$, Alberto Prina ${ }^{3}$, Mariana Pérez de la Torre ${ }^{2}$, \\ Alejandra Landau ${ }^{3}$ \\ ${ }^{1}$ Universidad de Buenos Aires, Facultad de Agronomía, Ciudad Autónoma de Buenos Aires, \\ Argentina. \\ ${ }^{2}$ Instituto Nacional de Tecnología Agropecuaria (INTA), Instituto de Floricultura., Buenos Aires, \\ Argentina. \\ ${ }^{3}$ Instituto Nacional de Tecnología Agropecuaria (INTA), Instituto de Genética "Ewald A. Favret", \\ Buenos Aires, Argentina. \\ Autor para correspondencia/ Corresponding autor, e-mail: bugallo@agro.uba.ar
}

Editado por /

Edited by:

Gabriela Albán

Recibido /

Received:

$7 / 08 / 2020$

Aceptado /

Accepted:

24/10/2020

Publicado en línea /

Published online:

01/04/2021

\section{X-rays treatments to obtain ornamental mutants in Salvia coccinea}

\section{Resumen}

El género Salvia posee gran valor ornamental, numerosas variedades en el mercado mundial y cuenta con alrededor de 900 especies, 19 de las cuales son nativas de Argentina. Salvia coccinea es una de la especies argentinas con mayor potencial ya que posee gran rusticidad, vigor y flores muy vistosas de un color rojo intenso. Sin embargo, al abordar el mejoramiento en esta especie, los problemas que se presentan son la dificultad para la hibridación interespecífica y la escasa variación fenotípica que expresa la especie. Para aumentar la variabilidad genética en programas de mejoramiento de plantas ornamentales, la aplicación de técnicas de inducción de mutaciones es ideal ya que puede modificar características de las flores, del follaje, del hábito de crecimiento y de variables fisiológicas. Con el objetivo de obtener variabilidad genética para la obtención de plantas ornamentales de $S$. coccinea, se aplicaron rayos $\mathrm{X}$ a semillas de un genotipo selecto con dosis de 100 a 600 Gy. Para evaluar el efecto producido, se estimó el área de los cotiledones por medio del análisis fotográfico, se calculó la dosis letal media (DL50) en la supervivencia de las plantas y se evaluaron los fenotipos obtenidos. La expansión de los cotiledones fue afectada en todos los tratamientos con aplicación de rayos $X$, mostrando un área menor que en el tratamiento control. La DL50 se estimó por regresión lineal, resultando en un valor de irradiación de 312 Gy. Entre los fenotipos mutantes se observaron deformaciones foliares, alteraciones en la pigmentación de las hojas (variegación, albinismo) y torsión en tallos. La selección de nuevas variedades se orientará a la obtención de plantas compactas, variegación de las hojas, novedades en forma y color de flores y esterilidad.

Palabras clave: DL50, Inducción de mutaciones, Mejoramiento genético, Plantas nativas, Plantas ornamentales. 


\begin{abstract}
The Salvia genus has great ornamental value, with numerous varieties on the world market. Around 900 species correspond to this genus, 19 of which are native to Argentina. Salvia coccinea is one of the Argentinean species with the greatest potential since it has great rusticity, vigor and very showy flowers of an intense red color. However, breeding in this species has two major constrains: a low variability and a difficult interspecific hybridization. To increase genetic variability in ornamental plants, mutation induction techniques are specially appropriated since they have great chances to modify characteristics of flowers, foliage, growth habit and physiological variables keeping unchanged most of the genetic background. In order to evaluate X-rays effects in S. coccinea, X-rays treatments were applied to seeds of a selected genotype in doses of 100 to $600 \mathrm{~Gy}$. To evaluate the effect produced, the area of the cotyledons was estimated by means of the analysis of photographs, the median lethal dose (LD50) in the survival of the plants was calculated, and the phenotypes obtained were evaluated. Cotyledon expansion was affected in all treatments with X-rays application, showing a smaller area than the control treatment. The LD50 was estimated using the formula obtained by linear regression, resulting in an irradiation value of $312 \mathrm{~Gy}$. Among the phenotypes modifications, leaf deformations, pigments alterations (variegation, albinism) and stem torsion were observed. The selection of mutants will focus on compact plants, leaf variegation, novelties in flower shape and color, and sterility.
\end{abstract}

Keywords: DL50, Induced mutations, Native plants, Ornamental plants, Plant mutation breeding.

\title{
INTRODUCCIÓN
}

Tanto en Argentina como en el resto de Sudamérica, los recursos genéticos nativos han sido poco explotados para el desarrollo de plantas ornamentales; sin embargo, el germoplasma proveniente de esta zona ha sido utilizado en el desarrollo de variedades por empresas internacionales. No obstante, a pesar de las inmensas ganancias que generan, los países de origen no reciben beneficio alguno [1].

El género Salvia (Lamiaceae) posee gran valor ornamental, tiene numerosas variedades en el mercado mundial y cuenta con alrededor de 900 especies de las cuales 19 son nativas de Argentina [2]. Salvia coccinea Buc'hoz ex Etl. es una de las especies argentinas con mayor potencial ya que posee gran rusticidad, vigor y flores muy vistosas de un color rojo intenso; sin embargo, al abordar el mejoramiento en esta especie, uno de los problemas que se presenta es la dificultad para la hibridación interespecífica y la escasa variabilidad fenotípica que presenta.

Para aumentar la variabilidad genética en programas de mejoramiento de plantas ornamentales, la aplicación de técnicas de inducción de mutaciones es especialmente apropiada. En estos programas, los objetivos principales son: modificar características de las flores como color, tamaño, morfología y fragancia; del follaje, como forma, tamaño y pigmentación; del hábito de crecimiento, como compacidad y ramificación; y de 
variables fisiológicas como cambios en la respuesta fotoperiódica, floración temprana, continua y tolerancia a estreses bióticos y abióticos [3]. Estos objetivos tienen altas probabilidades de ser alcanzados mediante la aplicación de técnicas de mutaciones inducidas manteniendo sin cambios la gran mayoría del contenido genético.

Con el objetivo de obtener variaciones fenotípicas en S. coccinea que permitan la obtención de nuevas variedades ornamentales, se aplicaron técnicas de inducción de mutaciones por rayos X. En este trabajo se efectuó la determinación de la dosis letal media (DL50) para esta especie, así como también la evaluación del efecto fenotípico temprano en la expansión de los cotiledones en plántulas y, posteriormente, el fenotipo en plantas adultas.

\section{MATERIALES Y MÉTODOS}

\section{Irradiación con rayos $X$}

El material vegetal empleado consistió en semillas de un genotipo selecto de $S$. coccinea proveniente de la colección in vivo del Instituto de Floricultura del INTA, Argentina.

Las semillas fueron irradiadas por medio de un equipo de rayos $X$ de tipo industrial a 120 kilovoltio (kV) y 15 miliamperio $(\mathrm{mA})$. Se aplicaron dosis incrementales de rayos $X$ en seis tratamientos: 100, 200, 300, 400, 500 y 600 Gy, más un control con semillas no irradiadas. Se irradiaron, por lo menos, 50 semillas por tratamiento. Para la estimación de la dosis letal media (DL50), se evaluó la cantidad de plantas adultas obtenidas respecto del tratamiento control, teniendo en cuenta la viabilidad de la semilla. El efecto de los tratamientos se analizó ajustando los datos a un modelo matemático de regresión lineal simple y, con la fórmula resultante, se obtuvo la DL50. La supervivencia de plantas entre las diferentes dosis y el control fueron contrastadas por medio de la prueba de diferencia de proporciones, realizados por medio del programa estadístico InfoStat 2009 [4].

\section{Germinación y evaluación de las plántulas}

Inmediatamente después de los tratamientos con rayos $X$, las semillas fueron embebidas en agua durante una hora luego de lo cual se les retiró el mucílago que exudan frotándolas en una toalla de papel, para evitar posibles barreras a la germinación (Figura 1). La siembra se realizó en bandejas plásticas alveoladas en un sustrato compuesto por turba, perlita y vermiculita en proporción 1:1:1 (Figura 1). Las bandejas de germinación se colocaron en invernáculo bajo un sistema de microaspersión hasta la emergencia de las hojas verdaderas.

Las plántulas fueron evaluadas en un estadio temprano, a los 20 días de la siembra. Para ello, se tomaron fotografías en vista superior de las bandejas alveoladas y se analizaron por medio del programa Image J versión 1.50i, estimando el área relativa de la superficie de los cotiledones en cada tratamiento. Las superficies de cotiledones entre cada tratamiento fueron contrastadas por medio de un ANOVA con comparaciones por BSS por medio del programa InfoStat 2009 [4]. 

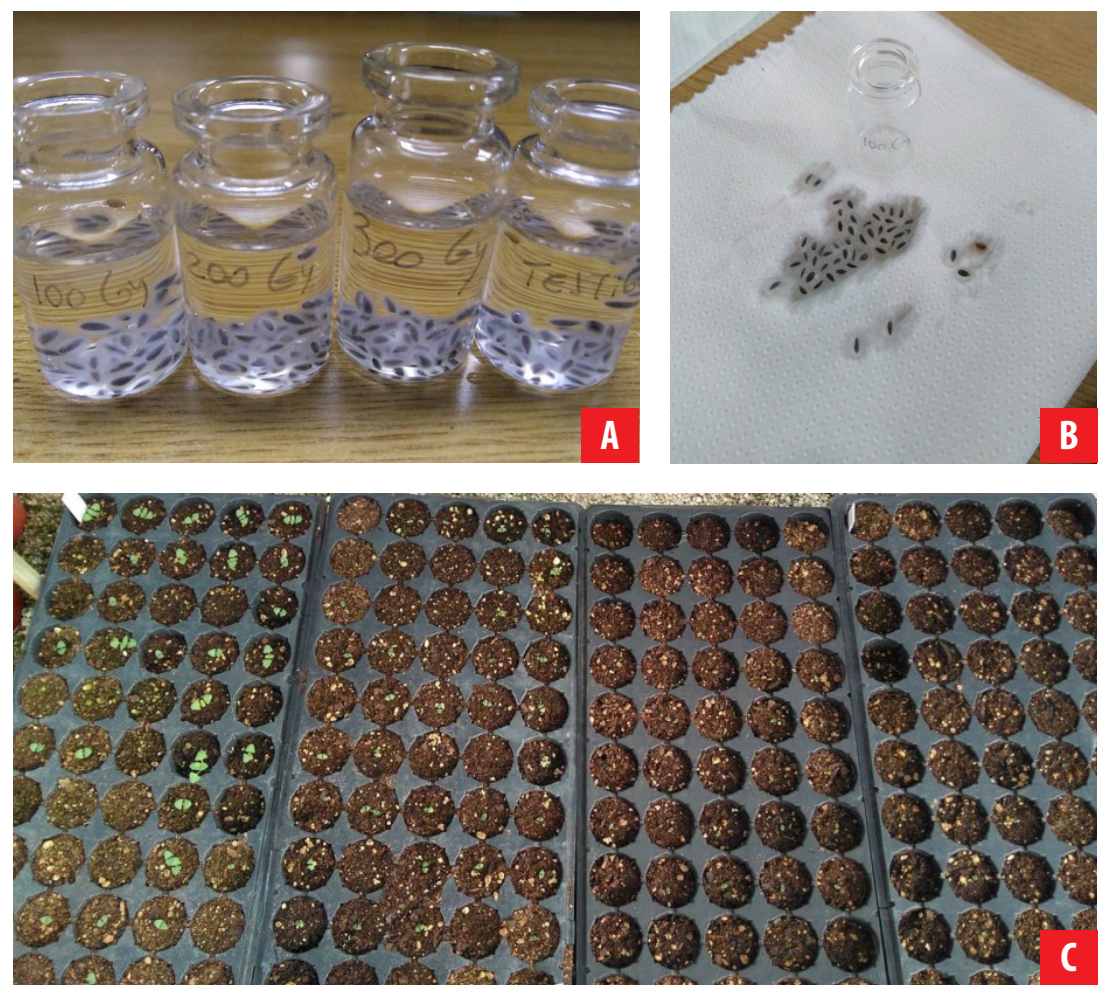

Figura 1: Tratamiento de semillas y siembra para la inducción de mutaciones por rayos $X$ en Salvia coccinea. A. Semillas en imbibición; B. limpieza del mucílago de las semillas previo a la siembra; $C$ : plántulas provenientes de la germinación de los tratamientos con dosis 0, 100, 200 y 300 Gy (de izquierda a derecha).

\section{Evaluación de plantas con fenotipos mutantes}

La evaluación de los fenotipos se realizó en los diferentes estadios de las plantas hasta la floración. Se realizaron comparaciones con las plantas del tratamiento testigo y se registraron fotográficamente los fenotipos aberrantes.

\section{RESULTADOS}

En un primer ensayo se aplicaron dosis de rayos X de 100, 200 y 300 Gy a semillas de S. coccinea y se estimó la viabilidad de las semillas del control en un 84\% (42 semillas germinadas de 50 totales). En un segundo ensayo con 400, 500 y 600 Gy, la viabilidad de las semillas del control fue de $96,3 \%$ (52 semillas germinadas de 54 totales). La viabilidad de las semillas de los tratamientos control fue utilizada para relativizar los porcentajes de supervivencia de los tratamientos con aplicación de rayos X (Tabla 1).

Por otro lado, se observaron resultados variables en cuanto a la supervivencia de plantas. Los porcentajes de formación de plantas adultas fueron desde un 85.7\% para el tratamiento con dosis de 100 Gy de rayos X hasta 0\% cuando la dosis ascendió 
a 600 Gy (Tabla 1). No se encontraron diferencias estadísticamente significativas en la supervivencia entre los tratamientos con 100, 200 y 300 Gy, mientras que se observaron diferencias en el número de plantas adultas sobrevivientes, cuando aumentaron las dosis por encima de 400Gy. La regresión lineal entre los porcentajes de supervivencia y las dosis de rayos $X$ aplicadas ajustó con un coeficiente de correlación $\mathrm{R}^{2}$ de 0.94 (Figura 2).

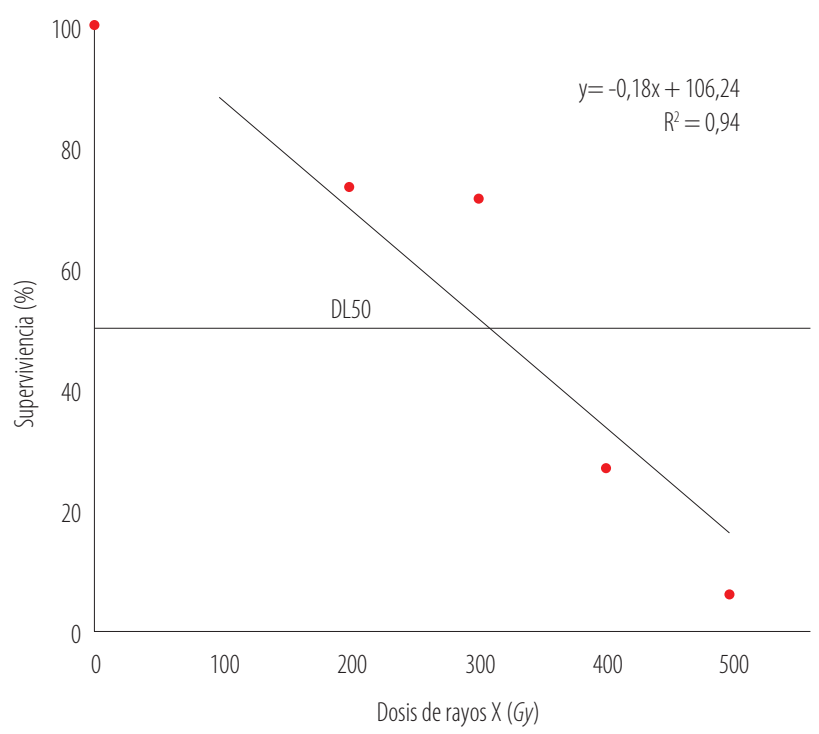

Figura 2. Porcentaje de supervivencia de las plantas en función de las dosis de rayos $X$ (Gy) aplicadas a las semillas (regresión lineal) y determinación de dosis letal media (DL50) en Salvia coccinea.

La dosis letal media (DL50) fue estimada por medio de la fórmula obtenida por regresión lineal, resultando en un valor de irradiación de 312 Gy (Figura 2).

El área media de los cotiledones mostró diferencias significativas entre los tratamientos con rayos $X$ y el control. En los cotiledones de las plántulas del primer ensayo, los tratamientos con 100 y 300 Gy no mostraron diferencias estadísticas entre sí; sin embargo, el tratamiento con 200 Gy mostró un tamaño significativamente menor. Los cotiledones de las plántulas del segundo ensayo, presentaron un tamaño menor a mayores dosis de rayos $X$, no mostrando diferencias estadísticas los tratados con 500 y 600 Gy. En la comparación entre todos los tratamientos, las dosis de 100, 300 y 400 Gy exhibieron un área de tamaños entre el 34.37 y el $38.16 \%$ respecto de los cotiledones control, mientras que los tratados con 200, 500 y 600 Gy, presentaron valores relativos entre un 22.62 y $31.09 \%$ (Tabla 1). 
Tabla 1: Supervivencia relativa total de plantas, porcentaje de supervivencia relativa y área de los cotiledones en plantas de Salvia coccinea irradiadas con rayos X. Letras distintas indican diferencias significativas entre los tratamientos.

\begin{tabular}{|c|c|c|c|}
\hline $\begin{array}{c}\text { Dosis de rayos } \\
\mathbf{X}(\mathbf{G y})\end{array}$ & $\begin{array}{c}\text { Supervivencia relativa de plantas } \\
\text { (plantas producidas/plantas } \\
\text { control) }\end{array}$ & $\begin{array}{c}\text { Supervivencia } \\
\text { relativa (\%) }\end{array}$ & $\begin{array}{c}\text { Área relativa media de } \\
\text { los cotiledones (\%) }\end{array}$ \\
\hline $0(100,200,300)$ & $42 / 42 \mathrm{a}$ & 100.0 & $100.0 \mathrm{a}$ \\
\hline 100 & $36 / 42 \mathrm{~b}(\mathrm{p} 0,0129)$ & 35.7 & $38.1 \mathrm{~b}$ \\
\hline 200 & $31 / 42 \mathrm{~b}(\mathrm{p} 0,1893)$ & 73.8 & $31.0 \mathrm{c}$ \\
\hline 300 & $30 / 42 \mathrm{~b}(\mathrm{p} 0,8125)$ & 71.4 & $34.4 \mathrm{~b}$ \\
\hline $5(400,500,600)$ & $52 / 52 \mathrm{a}$ & 100.0 & $100.0 \mathrm{a}$ \\
\hline 400 & $14 / 52 \mathrm{c}(\mathrm{p}<0,0001)$ & 26.9 & $36.6 \mathrm{~b}$ \\
\hline 500 & $3 / 52 \mathrm{~d}(\mathrm{p} 0,0037)$ & 5.7 & $27.4 \mathrm{c}$ \\
\hline 600 & $0 / 52 \mathrm{~d}(\mathrm{p} 0,1213)$ & 0 & $22.6 \mathrm{C}$ \\
\hline
\end{tabular}

En cuanto a la aparición de fenotipos aberrantes, éstos fueron observados a partir de 300 Gy. Entre ellos se observaron deformaciones foliares, alteraciones en la pigmentación de las hojas, variegación, albinismo y torsión en tallos (Figura 3). 

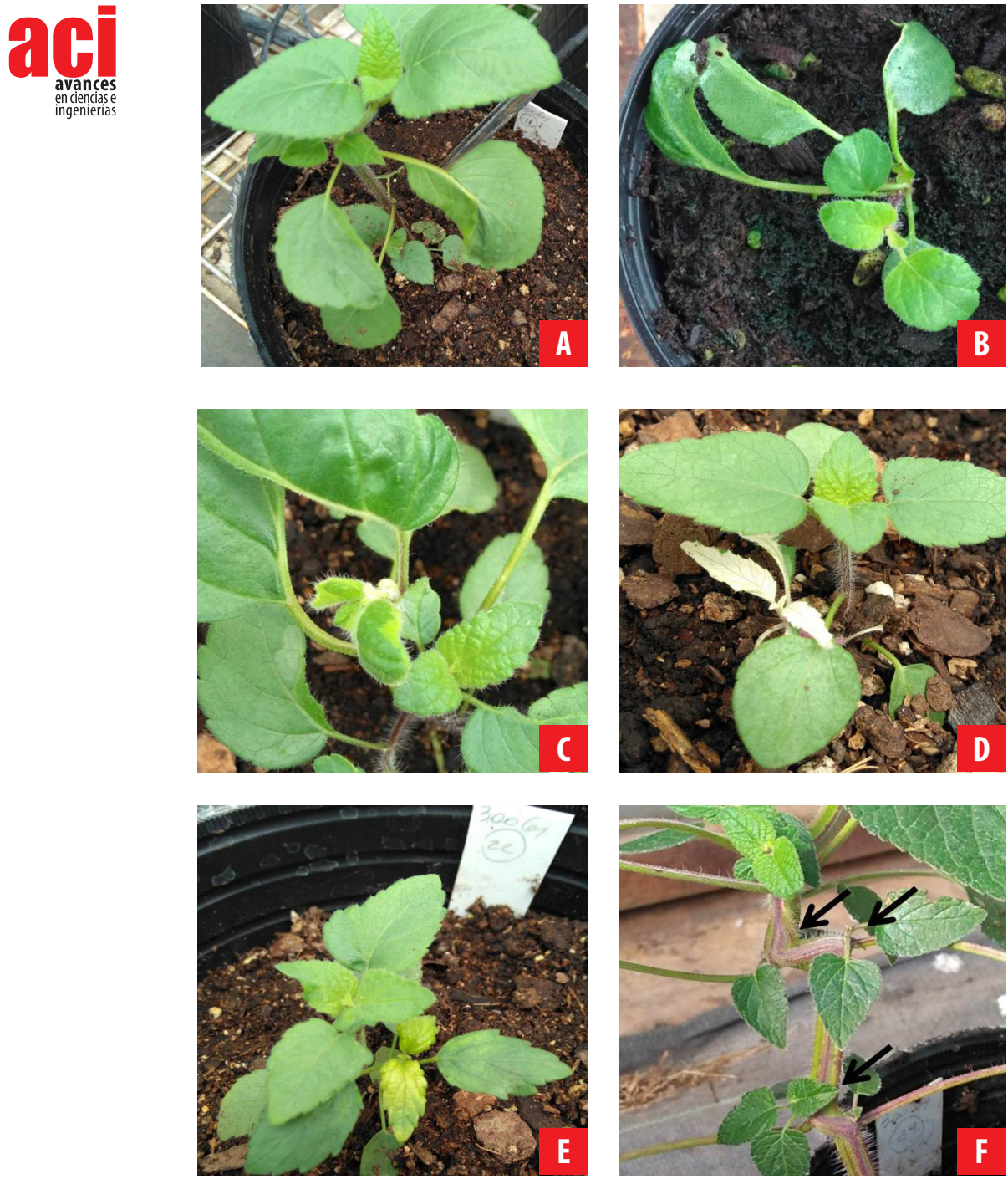

Figura 3: Efectos fenotípicos observados en plantas de Salvia coccinea provenientes de semillas irradiadas con rayos $X$. $A C, B$,: deformaciones foliares; $D$, E: cambios en la pigmentación de las hojas, variegación y albinismo; F: torsión en tallos.

\section{DISCUSIÓN}

La aplicación de técnicas de inducción de mutaciones ha sido ampliamente utilizada para la obtención de plantas ornamentales, con más de 700 variedades registradas [5]. Por estos métodos se han obtenido variedades en los géneros: Alstroemeria, Rosa, 
Chrysanthemum, Dahlia, Begonia y Dianthus, entre otros. Sin embargo, a la fecha no existe ninguna variedad de Salvia ornamental en dicho registro.

La dosis letal media (DL50) obtenida en este trabajo para S. coccinea fue de 312 Gy, la cual resultó alta comparada con la empleada para la obtención de mutantes a partir de semillas en algunas especies como Passiflora edulis y flavicarpa (125 Gy), Cucurbita maxima y Cucurbita moschata (160-190 Gy); pero resulta similar a la utilizada para arroz (345-423 Gy) [6-8]. Sin embargo, la DL50 estimada en especies crucíferas fue mucho mayor (400-800 Gy), siendo esta diferencia atribuida a diferencias en el volumen nuclear y el tamaño cromosómico [9]. El espesor y la textura del tegumento de la semilla también podrían alterar la DL50 [10].

Una de las ventajas del uso de inducción de mutaciones en el mejoramiento de plantas ornamentales propagadas vegetativamente es la posibilidad de seleccionar en la generación mutagenizada $M_{1}$ [3], ya que la propagación agámica, permite mantener mutantes al estado heterocigota. Por lo tanto, no es necesario llegar a la homocigosis para poder registrar la variedad. Esto también representa una oportunidad para el mejorador cuando puede alternarse con reproducción sexual, teniendo la posibilidad de seguir buscando variación mediante la autofecundación, así como también en la recombinación, mediante el cruzamiento entre mutantes diferentes.

El área media de la superficie de los cotiledones fue significativamente menor al control en todos los tratamientos ensayados. La reducción en el área de los cotiledones suele atribuirse al daño cromosósomico, dado que los rayos $X$ producen rupturas y rearreglos [11]. Esa hipótesis sugeriría que en los cotiledones de $S$. coccinea ocurriría división celular post-germinación. Sin embargo, deberán realizarse estudios para dilucidar cuál fue el mecanismo afectado.

Los fenotipos aberrantes obtenidos resultaron muy interesantes para los objetivos de mejoramiento. Algunas de las modificaciones encontradas en hojas como las variegaciones y cambios de pigmentación, así como la torsión de tallos (Figura 3) pueden proveer de la variabilidad requerida para generar una nueva variedad. Por otro lado, algunos de los fenotipos obtenidos mostraron indicios de que sus tejidos presentan un mosaico genético, siendo quimeras sectoriales o mericlinales (Figuras 3.c, d y e). En caso de resultar de interés, podría aislarse intentando enraizar la porción que presenta la mutación. La obtención de variantes sólidas en esta etapa, se atribuiría a la expresión de variación pre-existente no detectada en el fenotipo parental.

Existen diferentes metodologías para la inducción de mutaciones por rayos $X$. En este trabajo fueron aplicadas dosis agudas en una sola exposición a la radiación. Sin embargo, algunos autores han obtenido un mayor número de mutantes mediante una exposición fraccionada de la DL50 ya que, según afirman, permite al genoma recuperarse entre eventos de irradiación [12-15].

Si bien ya se obtuvieron fenotipos promisorios, el plan de mejoramiento continuará aplicando la DL 50 calculada para obtener un mayor número de mutantes. La aplicación de la radiación se repetirá como dosis aguda pero no se descarta la posibilidad de evaluar el efecto de dosis fraccionadas. También se planea realizar autofecundaciones y 
cruzamientos dirigidos entre mutantes. La selección de nuevas variedades se orientará a la obtención de plantas compactas, variegación de las hojas, novedades en forma y color de flores y esterilidad. Las plantas obtenidas a partir de los ensayos de inducción de mutaciones se encuentran actualmente en cultivo en el Instituto de Floricultura del INTA, Argentina, para continuar su evaluación.

\section{AGRADECIMIENTOS}

A Zulma Roa, quien mantiene con dedicación la colección de trabajo de Salvia en el Instituto de Floricultura del INTA.

\section{CONTRIBUCIONES DE LOS AUTORES}

Verónica Bugallo: concepción de la investigación; diseño de la metodología; redacción del manuscrito; adquisición, análisis e interpretación de los datos; estadísticas.

Gabriela Facciuto: concepción de la investigación, diseño de la metodología, redacción del manuscrito; adquisición, análisis e interpretación de los datos.

Vanina Brizuela: adquisición de los datos de laboratorio.

Alberto Prina: redacción del manuscrito, revisión crítica del contenido intelectual del manuscrito.

Mariana Pérez de la Torre: redacción del manuscrito, revisión crítica del contenido intelectual del manuscrito.

Alejandra Landau: adquisición de los datos de laboratorio, redacción del manuscrito, revisión crítica del contenido intelectual del manuscrito. 


\section{BIBLIOGRAFÍA}

[1] Bugallo, V., Pannunzio, M. J., Cardone, S. \& Facciuto, G. (2011). Breeding Advances in Passiflora Native to Argentina. Floriculture and Ornamental Biotechnology, Global Science Books, UK. 5 (1): 23-34. ISSN: 1749-0294.

[2] O'Leary, N. \& Moroni, P. (2016). Las especies de Salvia (Lamiaceae) para Argentina. Darwiniana nueva serie 4(1): 91 131.

[3] Schum, A. \& Preil, W. (1998). Induced mutations in ornamental plants. In: Jain, SM; Brar, DS; Ahloowalia, BS (Eds.), Somaclonal variation and induced mutation in crop improvement. Current plant science and Biotechnology in agriculture, vol. 32. Springer.

[4] Di Rienzo, J.A., Casanoves, F., Balzarini, M. G., Gonzalez, L., Tablada, M., Robledo, C.W. (2011). InfoStat. Universidad Nacional de Córdoba, Argentina. [5] FA0/IAEA. 2020. Mutant Variety Database. Recuperado de: http://mvd.iaea.org. Consultado en junio 2020

[6] Flores, P. S., \& Bruckner, C H. (2015). Radiossensibilidade de sementes e segmentos caulinares de maracujazeiroamarelo submetidos à radiação gama. Ciência Rural, 45(12), 2131-2136. https://doi.org/10.1590/0103$8478 \mathrm{cr} 20121274$

[7] Kurtar, E. S., Balkaya, A. \& Kandemir, D. (2017). Determination of semi-lethal (LD50) doses for mutation breeding of Winter squash (Cucurbita maxima Duch.) and pumpkin (Cucurbita moschata Duch.). Fresenius Environmental Bulletin 26(5): 3209-3216.

[8] Harding, S.S., Johnson, S. D., Taylor, D. R., Dixon, C. A. \& Turay, M. Y. (2012). Effect of Gamma Rays on Seed germination, seedling height, survival percentage and tiller production in some rice varieties cultivated in Sierra Leone. American Journal of Experimental Agriculture 2(2): 247-255.

[9] Gómez-Campo, C. \& Delgado, L. (1964). Radioresistance in crucifers. Radiation Botany 4(4):479-483.

[10] Olasupo, F. O., Ilori, C. 0., Forster, B. P. \& Bado, S. (2016). Mutagenic effects of gamma radiation on eight accession of Cowpea (Vigna unguiculata [L.] Walp.). American Journal of Plant Sciences, 7: 339-351.

[11] Ibrahim, R., Ahmad, Z., Salleh, S., Hassan, A. A. \& Ariffin, S. (2018). Mutation Breeding in Ornamentals. In:Ornamental Crops (pp. 175-211). Springer, Cham.

[12] Bologna, P., Soto, S., Coviella, M.A., Pannunzio, M.J. Facciuto, G. Prina A.R. \& Borja M. (2012). X-Rays as a tool for inducing variants of Calibrachoa. In: Proceedings XXVIIIth IHC-IS on Advances in Ornamentals, Landscape and Urban Horticulture. ISHS. Acta Horticulturae 937:941-946.

[13] Broertjes, C. (1972). Improvement of vegetatively propagated plants by ionizing radiation. In: Induced Mutations and Plant Improvement (Proceedings FA0/IAEA Study Group Metting, (pp.293-299). Buenos Aires, Argentina.

[14] Matsumura, S. (1961). Dose Rate and RBE for Radiation Induced Mutations in Plants. The Japanese Journal of Genetics VI, Suppl. 36:65-67.

[15] van Harten, A.M. (1998). Mutation Breeding-Theory and Practical Applications. Ed. Cambridge. 